\title{
ALGEBRAIC SURFACES AND IRRATIONAL CONNECTED SUMS OF FOUR MANIFOLDS
}

\author{
BY R. MANDELBAUM
}

Communicated by J. A. Wolf, January 1, 1977

Suppose $W$ is an irreducible nonsingular projective algebraic 3 -fold and $V$ a nonsingular hypersurface section of $W$. Denote by $V_{m}$ a nonsingular element of $|m V|$. Let $V_{1}, V_{m}, V_{m+1}$ be generic elements of $|V|,|m V|,|(m+1) V|$ respectively such that they have normal crossing in $W$. Let $S_{1 m}=V_{1} \cap V_{m}$ and $C=$ $V_{1} \cap V_{m} \cap V_{m+1}$. Then $S_{1 m}$ is a nonsingular curve of genus $g_{m}$ and $C$ is a collection of $N=m(m+1) V_{1}^{3}$ points on $S_{1 m}$. By [MM2] we find that

$$
V_{m+1} \text { is diffeomorphic to } \overline{V_{m}-T\left(S_{1 m}\right)} \cup_{\eta} \overline{V_{1}^{\prime}-T\left(S_{1 m}^{\prime}\right)}
$$

where $T\left(S_{1 m}\right)$ is a tubular neighborhood of $S_{1 m}$ in $V_{m}, V_{1}^{\prime}$ is $V_{1}$ blown up along $C, S_{1 m}^{\prime}$ is the strict image of $S_{1 m}$ in $V_{1}^{\prime}, T\left(S_{1 m}^{\prime}\right)$ is a tubular neighborhood of $S_{1 m}^{\prime}$ in $V_{1}^{\prime}$ and $\eta: \partial T\left(S_{1 m}\right) \rightarrow \partial T\left(S_{m}^{\prime}\right)$ is a bundle diffeomorphism.

Now $V_{1}^{\prime}$ is well known to be diffeomorphic to $V_{1} \# N\left(-C P^{2}\right)$ (the connected sum of $V_{1}$ and $N$ copies of $C P^{2}$ with opposite orientation from the usual). Thus in order to be able to inductively reduce questions about the structure of $V_{m}$ to ones about $V_{1}$ we must simplify the "irrational sum" (*) above.

The general question we can ask is then the following:

Suppose $M_{1}$ and $M_{2}$ are compact smooth 4-manifolds and $K$ is a connected $q$-complex embedded in $M_{i}$. Let $T_{i}$ be a regular neighborhood of $K$ in $M_{i}$ and let $\eta: \partial T_{1} \rightarrow \partial T_{2}$ be a diffeomorphism:

Set $V=\overline{M_{1}-T_{1}} \cup \overline{M_{2}-T_{2}}$. How can the topology of $V$ be described more simply in terms of those of $M_{1}$ and $M_{2}$ ?

In those notes we announce some results on this question for the case $q=$ 1,2 and indicate some applications to the topology of algebraic surfaces. Let $\approx$ be read as "is diffeomorphic to" and set $P=C P^{2}$ and $Q=-C P^{2}$. Then

THEOREM A. Let $M_{i}, T_{i}, \eta$ be as above and suppose $K$ is a wedge of $k 1$ spheres homotopic to zero in $M_{i}$. Suppose also $\eta$ is orientation-reversing (relative to the induced orientation on $T_{i}$ from $M_{i}$ in case $M_{i}$ is oriented) and identity-like (see definition below).

\section{Then either}

(a) $V \approx M_{1} \# M_{2} \# k\left(S^{2} \times S^{2}\right)$, or

(b) $V \approx M_{1} \# M_{2} \# k(P \# Q)$.

AMS (MOS) subject classifications (1970). Primary 57D55, 57 A15, $14 \mathrm{~J} 99$. 
(In case $M_{1} \# M_{2}$ is of odd type then by [W] both (a) and (b) hold.)

We note that $\partial T_{i}$ in the above theorem is a connected sum of $S^{1} \times S^{2}$ with a 'collapsing' map $c_{i}: \partial T_{i} \rightarrow K$ inherited from $\tilde{c}_{i}: T_{i} \rightarrow K$. We call a collection $\left\{e_{j}\right\}$ of 1 -spheres in $\partial T_{i}$ a 'nice geometrical basis' of $\partial T_{i}$ iff there exists a collection $\Sigma_{j}$ of 2 spheres in $\partial T_{i}$, generating $H_{2}\left(\partial T_{i} ; Z\right)$ such that $c\left(\Sigma_{j}\right)$ is a point and $e_{l} \cap \Sigma_{j}$ is empty if $l \neq j$ and is a transversal intersection in one point if $l=j$. Then a diffeomorphism $\eta: \partial T_{1} \rightarrow \partial T_{2}$ is called identity-like if it is isotopic to a map transforming 'nice geometrical basis' to 'nice geometrical basis'.

Now suppose $q=2$ and $K$ is an embedded 2-manifold. Then

THEOREM B. Let $M_{1}, M_{2}$ above be simply connected and suppose $K$ above is an embedded 2-manifold with the $T_{i}$ tubular neighborhoods of $K$ in $M_{i}$. Let $\eta: \partial T_{1} \rightarrow \partial T_{1}$ be a bundle-diffeomorphism.

Let $C$ be a fiber of $\partial T_{1} \rightarrow K$ considered as a loop in $V$. Let $k=$ minimal number of generators of $H_{1}(K, Z)$.

Then

(1) If $C$ is homotopic to zero in $V$ then either

$$
V \#\left(S^{2} \times S^{2}\right) \approx M_{1} \# M_{2} \# k\left(S^{2} \times S^{2}\right)
$$

or

$$
V \#(P \# Q) \approx M_{1} \# M_{2} \# k(P \# Q)
$$

with both alternatives holding if $M_{1} \# M_{2}$ is of odd type.

(2) If $M_{2}$ is obtained by blowing a manifold $N$ up by a o-process at a point of a submanifold $S$ whose strict image in $M_{2}$ is $K$ then $V \# P \approx M_{1} \# N \#$ $k(P \# Q)$.

Theorem B part (2) is of particular interest in applications to algebraic surfaces. In particular in the example we gave at the beginning of this note we can conclude that

$$
V_{m+1} \approx V_{m} \# V_{1} \#(N-1) Q \# 2 g\left(S_{1 m}\right)(P \# Q)
$$

(where $g\left(S_{1 m}\right)$ is the genus of the nonsingular curve $S_{m}$ ). In particular if we take $W=C P^{3}$ and $V_{1}$ a hyperplane of $W$ then (**) above implies the main theorem of [MM1], which states that if $V$ is any nonsingular hypersurface of $C P^{3}$ then $V \# P$ is diffeomorphic to a connected sum of $P$ 's and $Q$ 's.

We can apply Theorem $B$ to get the following type of result.

Let us call a simply connected 4-manifold $M$ completely decomposable iff for some integers $k, M \approx k P \# l Q$.

Then we have

THEOREM C. Suppose $X$ is a nonsingular algebraic surface such that $X \# P$ is completely decomposable. Let $C$ be an irreducible nonsingular hypersurface 
section of $X$. Let $W \stackrel{\pi}{\rightarrow} X$ be the projective bundle over $X$ obtained by compactifying the line bundle $E=[k C](k \geqslant 0)$.

Then

(1) If $V$ is an irreducible, nonsingular hypersurface section of $W$ then $V$ \# $P$ is completely. decomposable.

(2) If $V$ is a nonsingular irreducible subvariety of $W$ such that $\pi \mid V \rightarrow X$ is an $m$-fold branched cover of $X$ then $V \# P$ is completely decomposable.

(3) If $V$ is a nonsingular $k$-fold cyclic branched cover of $X$ with branch locus $R$ linearly equivalent to $k C$ for some $k>0$ then $V \# P$ is completely decomposable.

In particular the nonsingular 'double-planes' (i.e., 2-fold branched covers of $C P^{2}$ ) are all completely decomposable after taking their connected sum with $C P^{2}$.

\section{REFERENCES}

[MM1] R. Mandelbaum and B. Moishezon, On the topological structure of non-singular algebraic surfaces in $C P^{3}$, Topology 15 (1976), 23-40.

[MM2] - On the topology of algebraic surfaces, Topology (to appear).

IW] C. T. C. Wall, Diffeomorphisms of 4-manifolds, J. London Math. Soc. 39 (1964), 131-140. MR 29 \#626.

WEIZMANN INSTITUTE OF SCIENCE, REHOVOT, ISRAEL

Current address: School of Mathematics, Institute for Advanced Study, Princeton, New Jersey 08540 